\title{
Range-resolved signal processing for fibre segment interferometry applied to dynamic long-gauge length strain sensing
}

\author{
Thomas Kissinger, Ricardo Correia, Thomas O. H. Charrett, Stephen W. James and Ralph P. Tatam* \\ Engineering Photonics, Cranfield University, Cranfield, MK43 0AL, United Kingdom \\ *r.p.tatam@cranfield.ac.uk
}

\begin{abstract}
A range-resolved interferometric signal processing technique using sinusoidal optical frequency modulation is applied to fibre segment interferometry. Here, six optical fibre segments of gauge length $12.5 \mathrm{~cm}$ are used as interferometric strain sensors and are formed between seven weak, broadband fibre Bragg gratings, acting as in-fibre partial reflectors. In a very simple and cost-effective optical setup using injection current modulation of a laser diode source, interferometric measurement of acoustic wave propagation in a metal rod is used to demonstrate the capabilities of the technique.
\end{abstract}

Keywords: Interferometry, Fibre Strain Sensing, Signal Processing, Fibre Segment Interferometry

\section{INTRODUCTION}

Range-resolved interferometric signal processing techniques based on optical frequency modulation exploit the proportionality of the induced phase excursion on the optical path difference (OPD) to separate return signals based on their range. Previous approaches include the well-known pseudoheterodyne technique [1, 2], using linear or sawtooth optical frequency modulation, which has been employed for range-based multiplexing of interferometric fibre-coupled displacement sensors [2], or sinusoidal optical frequency modulation, which contains only one frequency component and is thus simpler to implement [3-5]. For the latter, the sinusoidal phase-generated carrier method [3] has been used widely, but is not range-resolved and cannot multiplex interferometers using a single source and photo detector. Using sinusoidal frequency modulation in conjunction with rectangular gating $[4,5]$, however, allows interferometric sensors to be multiplexed [5]. In general, previous linear and sinusoidal range-resolved interferometric techniques [2, 5] have required the use of integer ratios between the OPDs of constituent interferometers for proper operation, leading to high positioning tolerances as well as leaving measurements susceptible to crosstalk from parasitic or unintended signal sources that do not adhere to the integer OPD ratio requirement. In contrast, a recently developed sinusoidal technique [6], using a smooth window function instead of rectangular gating, permits continuously variable OPD placement of the signal sources, with no apparent penalty in linear operation or crosstalk as long as a minimum OPD separation between the constituent interferometers is observed, greatly increasing the practicality of the approach.

In this work, this novel range-resolved signal processing approach [6] is applied to fibre segment interferometry (FSI), where optical fibre segments are formed between in-fibre partial reflectors and are interrogated in a low-finesse FabryPerot setup. Phase measurements from each segment are then obtained by subtraction of the partial reflector signals, resulting in an array of long-gauge length interferometric sensors that integrate any OPD-induced phase changes due to strain, temperature etc. This approach was developed originally for hydrophone applications [7] and past implementations have been based mainly on time-division multiplexing [7-9] or code-division multiplexing [10]. When this new signal processing approach [6] is applied to FSI, the setup of the interrogation unit is simplified considerably, employing no external modulator, and the minimum resolvable fibre segment length improves significantly, reducing from many metres, as reported in prior work [7-10], to tens of centimetres as demonstrated here. Furthermore, the use of low-reflectivity, broadband fibre Bragg gratings (FBGs) [9], all inscribed at the same wavelength, to act as partial infibre reflectors eliminates the need for splices $[7,10]$ or couplers $[8]$ to define the segments within the sensing fibre.

\section{SIGNAL PROCESSING}

The novel signal processing approach [6] employed in this work uses a sinusoidal optical frequency modulation waveform of modulation frequency $f_{\text {mod. }}$. As illustrated in Fig. 1(a), this waveform has a peak optical frequency excursion $\Delta f$ and is centred around optical frequency $f_{0}$. For an interferometer with non-zero OPD this results in a 
corresponding sinusoidal phase modulation waveform of peak phase excursion A, producing a characteristic interferometric signal on the photo detector. For several constituent interferometers the characteristic interferometric signals are superimposed, as visible in the example photo detector signal $U(t)$ in Fig. 1(b), calculated for two constituent interferometers with OPD's resulting in peak phase excursions of $A_{1}=50 \mathrm{rad}$ and $A_{2}=100 \mathrm{rad}$. The key step in the novel signal processing approach [6] is the multiplicative application of a smooth window function to the photo detector signal $U(t)$. In this work, a window function $W(t)$, plotted in Fig. 1(b), containing two individual Gaussian windows that are centred on the two points of inflection of the sinusoidal frequency modulation waveform that is used.
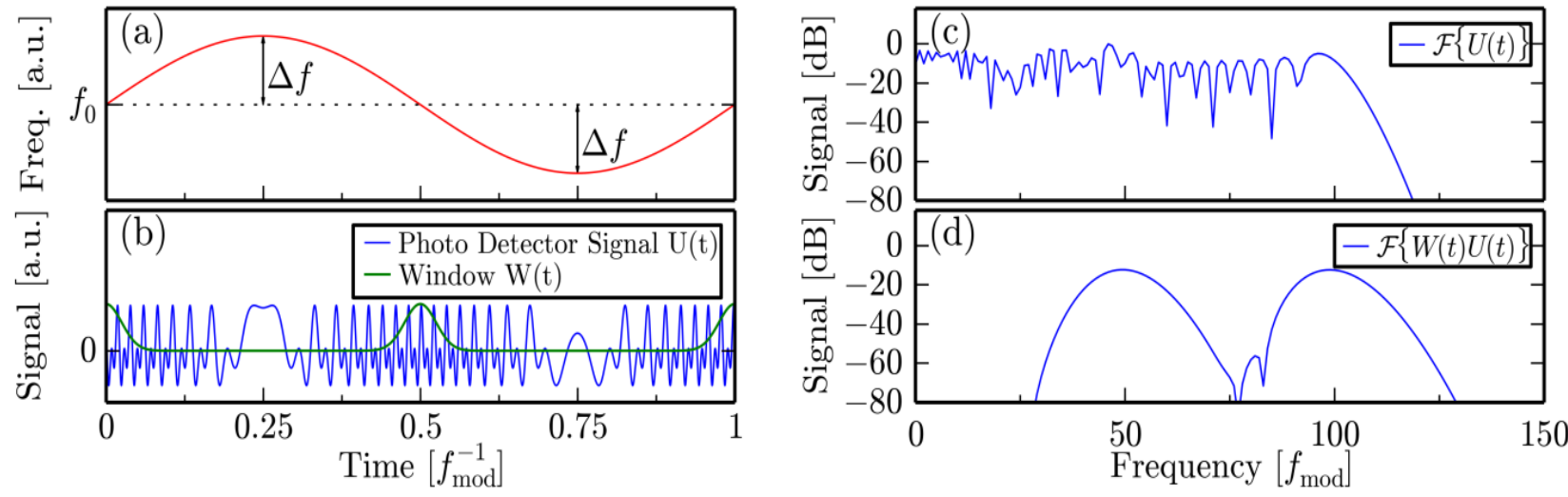

Figure 1. Illustration of the general principles of the signal processing approach. A sinusoidal optical frequency modulation (a) results in a characteristic interferometric signal in the photo detector signal $U(t)$ that can be superimposed for several interferometers, shown in (b) for interferometers with OPD's resulting in peak phase excursions $A_{1}=50 \operatorname{rad}$ and $A_{2}=$ $100 \mathrm{rad}$, along with the window function $W(t)$. (c) and (d) compare the Fourier spectrum of $U(t)$ without and with the application of $W(t)$, respectively, highlighting the effect of $W(t)$ in separating signals into distinct frequency bands.

The effect of the multiplication of $W(t)$ becomes obvious when comparing Fig. 1(c) and Fig. 1(d). Here, the Fourier spectrum of $U(t)$ on its own can be seen in Fig. 1(c) to be mostly flat, with all Bessel frequency components present. In contrast, the Fourier spectrum of the windowed photo detector signal $W(t) U(t)$ shows a clearly visible separation into two distinct peak regions. Here, the peak around $50 f_{\text {mod }}$ is found to correspond to the interferometer with peak phase excursion $A_{1}$, while the peak around $100 f_{\text {mod }}$ is found to correspond to the interferometer with peak phase excursion $A_{2}$. This highlights the key principle of the technique that the application of the window function in the time domain results in the separation of the signal components of constituent interferometers into distinct frequency bands. In contrast to earlier work [5], where rectangular gating was used instead of a smooth window function, the peak regions in Fig. 1(d) do not show any side lobe components, where the avoidance of crosstalk from side lobe components had previously lead to the discussed restriction to integer OPD that is absent in this novel approach. A further improvement results from the use of a time-variant complex demodulation carrier [6] that resembles approximately the expected complex characteristic interferometric signal at a given OPD of the constituent interferometer and that can be implemented easily in digital signal processing. In contrast to the use of a fixed frequency carrier in prior works $[4,5]$ this reduces non-idealities arising from the approximation of the exact sinusoidal phase waveform by a single frequency carrier. Together, both of these measures result in a range-resolved interferometric signal processing technique that is highly linear and flexible and which furthermore can be made tolerant to non-idealities resulting from the use of laser injection current modulation, such as associated laser intensity modulation and nonlinearities in frequency modulation [6].

\section{EXPERIMENTS}

The experimental setup used in this work is illustrated in Fig. 2. Here the FSI sensing fibre, consisting of six fibre segments (S1 to S6) formed using in-fibre FBG partial reflectors spaced at $12.5 \mathrm{~cm}$ intervals, is attached to a freely suspended stainless steel rod. The interferometric reference is taken from the fibre tip reflection, providing a simple, selfreferencing configuration with complete down-lead insensitivity. The optical setup consists of a laser diode, modulated by a sinusoidal injection current waveform, a circulator and a photo detector connected using regular single-mode fibre. The laser diode is a cost-effective DFB-type laser $(\lambda=1550 \mathrm{~nm})$, originating from telecom applications and all modulation and demodulation is carried out synchronously using low-cost field programmable gate array (FPGA)-based processing at a digital sample rate of $150 \mathrm{MHz}$. The FBG partial reflectors used, manufactured in-house, were very broadband $(>5 \mathrm{~nm})$ and all inscribed at the same centre wavelength to ensure that the FBGs would return a 


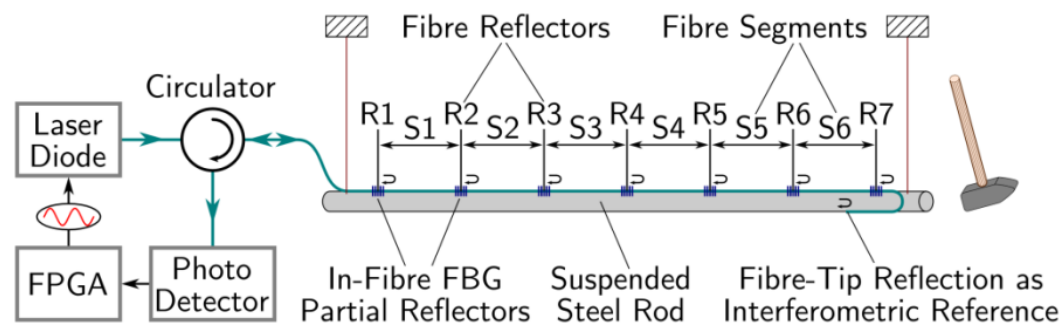

Figure 2. Experimental setup used to measure acoustic wave propagation after a hammer strike. Sinusoidally frequency modulated light from a laser diode is guided to an optical fibre mounted on a freely suspended steel rod, where seven partial reflectors R1 to R7 form six fibre segments $\mathrm{S} 1$ to $\mathrm{S} 6$ of gauge length $12.5 \mathrm{~cm}$. The light from the reflectors, along with the interferometric reference provided by the fibre tip, is guided to the photo detector and the signals demodulated in the FPGA.

signal under all practical strain and temperature conditions. The reflectivity was low $(<0.01 \%)$, but the heterodyne gain from the interferometric reference ensured that the return signals were sufficiently strong for the measurement to remain in laser-phase-noise-limited operation. The data rate of $98 \mathrm{kHz}$ in the current implementation allows an unambiguous interferometric fringe rate of $45 \mathrm{kHz}$, corresponding to a maximum strain change rate over the whole array of six segments of approximately $40 \mathrm{~m} \epsilon \cdot \mathrm{s}^{-1}$. This could be improved through the use of a higher modulation frequency $f_{\text {mod }}$. The minimum resolvable fibre segment length is a property of the peak frequency excursion $\Delta f$ achievable by the laser.
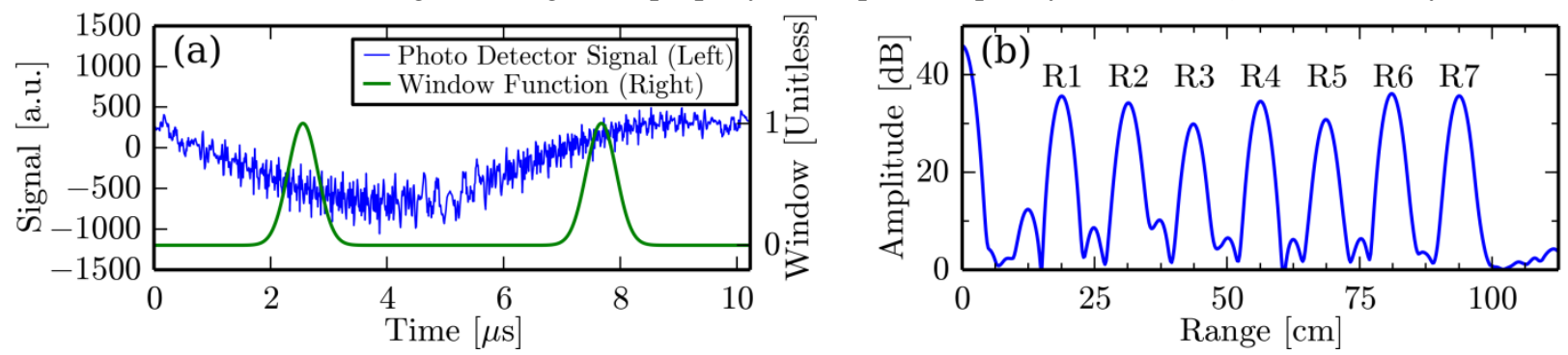

Figure 3. (a) shows a captured photo detector signal over one modulation period along with the appropriately shifted window function on the secondary y-axis. (b) then plots the return signals after demodulation with the described timevariant carrier [6] as a function of range, with the peaks labeled according to respective originating reflector.

A typical photo detector signal resulting from this arrangement, which also displays some of the undesired influences, such as intensity modulation, and the appropriately temporally delayed window function that is used, are plotted in Fig. 3(a). After demodulation with the time-variant carrier and procedures to correct modulation non-idealities and delays described in [6], the range dependency of the return signals can be calculated and is shown in Fig. 3(b). Here, the seven peaks that correspond to reflectors R1 to R7 are marked. Demodulation at these ranges then results in the reflector signals, with the segment signals S1 to S6 resulting from a numerical subtraction of the bounding reflector phase signals.

\section{RESULTS AND DISCUSSION}

In example measurements using the setup described in Fig. 2, the propagation of an acoustic excitation through the stainless steel rod resulting from a hammer impact can be observed in Fig. 4. Here, a range-dependent delay of the initial

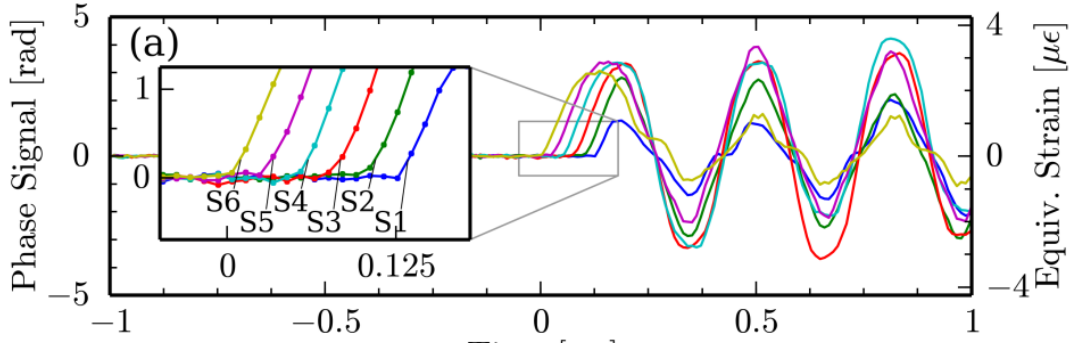

Time $[\mathrm{ms}]$

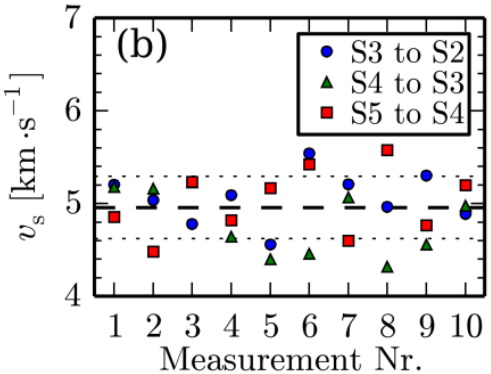

Figure 4. (a) plots segment signals S1 to S6 at the time of the hammer impact, with the inset showing the delayed onset of the initial signal rises and the secondary $\mathrm{y}$-axis on the main plot in equivalent strain units [10] for a $12.5 \mathrm{~cm}$ fibre segment.

(b) then shows the speed-of-sound $v_{\mathrm{s}}$ corresponding to the extracted delay times, where only the central segments are used due to edge effects and with the mean and standard deviation marked by the dashed and dotted lines, respectively. 
signal rise is clearly visible in the inset. By evaluating this delay over 10 repeats of this experiment, where only the central segments S2 to S5 were used to exclude edge effects, the speed-of-sound $v_{\mathrm{S}}$ in the rod was determined as $4.96 \pm 0.34 \mathrm{~km} \cdot \mathrm{s}^{-1}$, which is within the error limits of the expected value of $4.91 \mathrm{~km} \cdot \mathrm{s}^{-1}$ for a longitudinal acoustic wave in a thin stainless steel (Type 316) rod calculated using [11]. There is a mostly flat noise floor in the segment data, with typical noise levels of $0.2 \mathrm{mrad} \cdot \mathrm{Hz}^{-0.5}$, which is mainly attributed to the phase noise properties of DFB type laser sources [12]. These phase noise levels equate to a dynamic strain sensitivities [10] of $0.2 \mathrm{n \varepsilon} \cdot \mathrm{Hz}^{-0.5}$ for a $12.5 \mathrm{~cm}$ fibre segment, which compare well to widely used FBG strain sensing techniques with dynamic sensitivities of several $\mathrm{n} \varepsilon \cdot \mathrm{Hz}^{-0.5}$ [13], especially considering the high data rates of at least $98 \mathrm{kHz}$ that are possible using this approach. It is also worth noting that in FSI, strain data is integrated over the whole gauge length of the sensor, reducing the impact of localized disturbances [14] and leaving no sensing gaps, which could be advantageous in damage detection applications or vibration based condition monitoring [15]. Furthermore, there are no moving parts or components that require precise alignment, promising very robust interrogation units that could also be integrated easily into moving frames of reference.

\section{CONCLUSION}

A novel range-resolved interferometric signal processing technique has been applied to the multiplexing of fibre optic long-gauge length strain sensors. Using a very simple optical setup, measurements of six fibre segments of length 12.5 $\mathrm{cm}$, formed between seven FBGs acting as in-fibre partial reflectors, were demonstrated. Phase noise levels of $0.2 \mathrm{mrad} \cdot \mathrm{Hz}^{-0.5}$, at data rates of $98 \mathrm{kHz}$, were achieved with equipment cost in this prototype totaling below $\$ 5 \mathrm{k}$.

\section{ACKNOWLEDGEMENTS}

The authors acknowledge the support of the Engineering and Physical Sciences Research Council (EPSRC) UK, via grant EP/H02252X/1 and EP/G033900/1.

\section{REFERENCES}

[1] Jackson, D. A., Kersey, A. D., Corke, M. and Jones, J. D. C., "Pseudoheterodyne detection scheme for optical interferometers," Electron. Lett. 18, 1081-1083 (1982).

[2] Zheng, J., "Triple-sensor multiplexed frequency-modulated continuous-wave interferometric fiber-optic displacement sensor," Appl. Opt. 46, 2189-2196 (2007).

[3] Dandridge, A., Tveten, A. B., Giallorenzi, T. G., "Homodyne demodulation scheme for fiber optic sensors using phase generated carrier," IEEE J. Quantum Electron. 18, 1647-1653 (1982).

[4] Kersey, A.D., Lewin, A.C. and Jackson, D.A., "Pseudo-heterodyne detection scheme for the fibre gyroscope," Electron. Lett. 20, 368-370 (1984).

[5] Sakai, I., Youngquist, R. and Parry, G., "Multiplexing of optical fiber sensors using a frequency-modulated source and gated output," J. Lightwave Technol. 5, 932-940 (1987).

[6] Kissinger, T., Charrett, T. O. H. and Tatam, R. P., "Range-resolved interferometric signal processing using sinusoidal optical frequency modulation," Opt. Express, in press (2015).

[7] Dakin, J. P., Wade, C. A., Henning, M., "Novel optical fibre hydrophone array using a single laser source and detector," Electron. Lett. 20, 53-54 (1984).

[8] Cranch, G. A., Nash, P. J., Kirkendall, C. K., "Large-scale remotely interrogated arrays of fiber-optic interferometric sensors for underwater acoustic applications," IEEE Sensors J. 3, 19-30 (2003).

[9] Okawara, C. and Saijyou, K., "Fiber optic interferometric hydrophone using fiber Bragg grating with time division multiplexing," Acoust. Sci. Technol. 28, 39-42 (2007).

[10] Kissinger, T., Charrett, T. O. H. and Tatam, R. P., "Fibre segment interferometry using code-division multiplexed optical signal processing for strain sensing applications," Meas. Sci. Technol. 24, 094011 (2013).

[11] Graff, K. F., [Wave motion in elastic solids], Courier Dover Publications (1975).

[12] Bartolo, R. E., Tveten, A. B. and Kirkendall, C. K., "The quest for inexpensive, compact, low phase noise laser sources for fiber optic sensing applications," Proc. SPIE 7503, 750370 (2009).

[13] Lee, B., "Review of the present status of optical fiber sensors," Opt. Fiber Technol. 9, 57-79 (2003).

[14] Glisic, B., "Influence of the gauge length on the accuracy of long-gauge sensors employed in monitoring of prismatic beams," Meas. Sci. Technol. 22, 035206 (2011).

[15] Montalvao, D., Mendes Maia, N. M. and Relógio Ribeiro, A. M., "A review of vibration-based structural health monitoring with special emphasis on composite materials," Shock Vibration Digest 38, 295-326 (2006). 
$2015-11$

Range-resolved signal processing for fibre segment interferometry applied to dynamic long-gauge length strain sensing

\section{Kissinger, Thomas}

SPIE

Thomas Kissinger ; Ricardo Correia ; Thomas O. H. Charrett ; Stephen W. James and Ralph P. Tatam. Range-resolved signal processing for fibre segment interferometry applied to dynamic long-gauge length strain sensing. Proc. SPIE 9634, 24th International Conference on Optical Fibre Sensors, 96341Q, September 28, 2015.

https://dspace.lib.cranfield.ac.uk/handle/1826/9609

Downloaded from Cranfield Library Services E-Repository 